\title{
Extending the range of particle densities observed by GRAPES-3
}

\author{
A. Chandra ${ }^{*}, b$, S. Ahmad ${ }^{a, c}$, K.P. Arunbabu ${ }^{a, b}$, S.R. Dugad ${ }^{a, b}$, S.K. Gupta ${ }^{a, b}$, B. \\ HariHaran $^{a, b}$, Y. Hayashi ${ }^{a, d}$, P. Jagadeesan ${ }^{a, b}$, A. Jain ${ }^{a, b}$, V.B. Jhansi ${ }^{a, b}$, S. \\ Kawakami $^{a, d}$, H. Kojima ${ }^{a, e}$, P.K. Mohanty ${ }^{a, b}$, S.D. Morris ${ }^{a, b}$, P.K. Nayak ${ }^{a, b}$, A. \\ Oshima $^{a, f}$, B.S. Rao ${ }^{a, b}$, L.V. Reddy ${ }^{a, b}$, S. Shibata ${ }^{a, f}$, M. Zuberi ${ }^{a, b}$ \\ ${ }^{a}$ GRAPES-3 Experiment, Cosmic Ray Laboratory, Raj Bhavan, Ooty 643 001, India. \\ ${ }^{b}$ Tata Institute of Fundamental Research, Homi Bhabha Road, Mumbai 400 005, India. \\ ${ }^{c}$ Department of Physics, Aligarh Muslim University, Aligarh 202 002, India. \\ ${ }^{d}$ Graduate School of Science, Osaka City University, Osaka 558-8585, Japan \\ ${ }^{e}$ Faculty of Engineering, Aichi Insitute of Technology, Toyota City, Aichi 470-0392, Japan \\ ${ }^{f}$ College of Engineering, Chubu University, Kasugai, Aichi 487-8501, Japan \\ E-mail: anuj.hepegmail.com
}

The GRAPES-3 experiment is a unique facility to study cosmic ray energy spectrum and composition with high precision. It consists an array of 400 plastic scinitillation detectors and a muon telescope of 3712 proportional counters to study extensive air shower (EAS) phenomenon around knee ( $\gtrsim 10^{15} \mathrm{eV}$ ). Study of energy spectrum and composition of primary cosmic rays (PCR) can improve the understanding about the nature of the sources accelerating PCRs to energies $\gtrsim 10^{15}$ $\mathrm{eV}$ which may be studied by using high statistics data produced by GRAPES-3. Measurement of particle densities $\left(\gtrsim 5000 \mathrm{~m}^{-2}\right.$ ) is achieved by viewing each scintillator with two photomultiplier tubes (PMTs). The results obtained till date will be presented at the conference.

35th International Cosmic Ray Conference - ICRC2017

10-20 July, 2017

Bexco, Busan, Korea

${ }^{*}$ Speaker. 


\section{Introduction}

The detailed measurement and studies of primary cosmic rays (PCR) may reveal some clues to understand the acceleration and propagation of the cosmic rays in various astrophysical environments such as supernovae explosions, pulsars, relativistic jets, active galactic nuclei etc. The energy spectrum of cosmic rays exhibits a specific power law variation over a long range of energies from $\sim 10^{9}$ to $\sim 10^{20} \mathrm{eV}$ and exhibits two salient features known as knee at $\sim 3 \times 10^{15} \mathrm{eV}$ and ankle at $\sim 3 \times 10^{18} \mathrm{eV}$. Since the charged cosmic rays are hindered with the chaotic inter-galactic and interstellar magnetic fields, therefore, they are deflected from their original direction of origin and get modulated. It is believed that the investigations of cosmic rays through the elemental compositions around these two features may give us clues about their source of origin and the modulations.

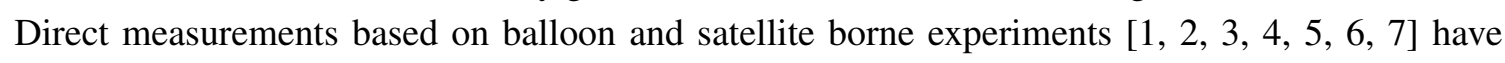
contributed to study of PCR up to $\sim 10^{15} \mathrm{eV}$. Beyond this energy, the ground based experiments are used due to their longer exposure time and larger area. The inter-detector separation plays a crucial role for higher energy thresholds for most of the extensive air shower (EAS) arrays [ [ ]].

Since the number of secondary particles produced in the EAS is an increasing function of PCR energy [9], detector design should allow to record higher particle densities. To extend the observation of particle densities to higher energy, the GRAPES-3 experiment has deployed plastic scintillator detectors equipped with two photo multiplier tubes (PMTs). On the other hand, the compositions of PCR can be studied by discriminating the showers on the basis of muon content with respect to the core of the showers.

\section{The GRAPES-3 Experiment}

The GRAPES-3 (Gamma Ray Astronomy at PeV Energies Phase-3) experiment is a high density EAS array located at Ooty in India $\left(11.4^{\circ} \mathrm{N}, 76.7^{\circ} \mathrm{E}, 2200 \mathrm{~m}\right.$ above m.s.l). Currently, it comprises 400 plastic scintillator detectors[ए]] of each of $1 \mathrm{~m}^{2}$ area and the layout is visible in Fig. س. They are installed in a hexagonal configuration with $8 \mathrm{~m}$ inter-detector separation. This combination of $8 \mathrm{~m}$ separation with the altitude at Ooty $\left(\sim 800 \mathrm{~g} . \mathrm{cm}^{-2}\right)$ can push the threshold of shower triggering energy as low as $\sim 30 \mathrm{TeV}[\mathrm{W}]$ in order to provide an overlap with direct measurements. In addition, the muon detector consists of 16 modules with each module $35 \mathrm{~m}^{2}$ in area using 4 layers of proportional counters. Each layer consists of 58 proportional counters, each $6 \mathrm{~m}$ long with $10 \times 10 \mathrm{~cm}^{2}$ cross-sectional area. Alternate counter layers are arranged orthogonally to permit good tracking of muons. A thick layer of absorber (concrete $\sim 550 \mathrm{~g} . \mathrm{cm}^{-2}$ ) above the $1^{\text {st }}$ counter layer provides a threshold to detect muons above $\geq 1 \mathrm{GeV}$. A cross-section of two adjacent muon detector modules is shown in Fig. $[\mathrm{Zb}$ and 4 such modules are placed in one common station. The four stations are visible in Fig. 2a.

\subsection{Types of Scintillator Detectors}

Scintillator detectors which have been developed in house at Ooty and are followed by various R\&D activities can be categorized as follows:

- Cone Detectors: The cone type of scintillator detectors are the earliest configuration, being used in the GRAPES-3 experiment[[0]]. All of them are of $1 \mathrm{~m}^{2}$ each and having four 50 


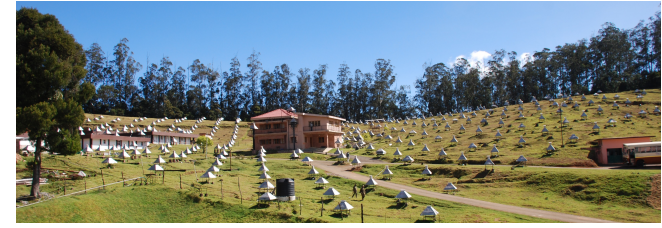

(a) Array Layout

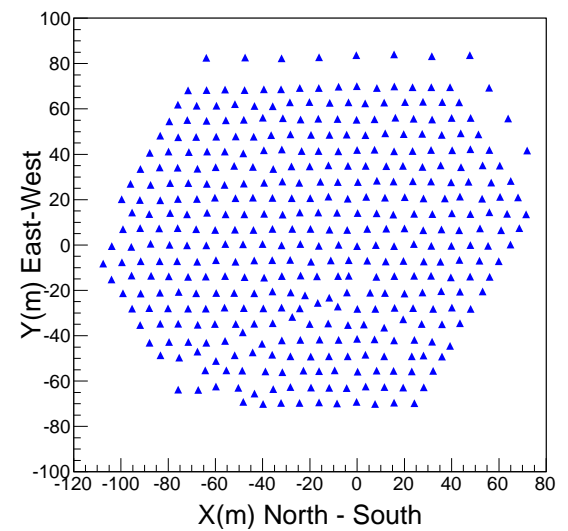

(b) Array Map

Figure 1: The GRAPES-3 EAS array deployed in hexagonal rings over $25,000 \mathrm{~m}^{2}$ area.

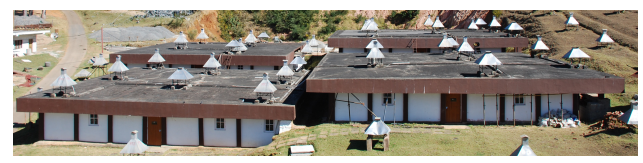

(a) 4 Muon Stations

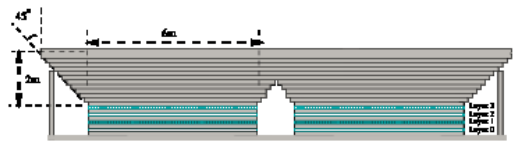

(b) Cross sectional view of module inside one station

Figure 2: The GRAPES-3 Muon Detectors

$\times 50 \mathrm{~cm}^{2}$ area and $5 \mathrm{~cm}$ thick plastic scintallator blocks placed on the base of a trapezoidal shaped aluminum cone. A photo multiplier tube (PMT) of $5 \mathrm{~cm}$ diameter window is placed at $60 \mathrm{~cm}$ above the surface of scintillator blocks facing towards scintillators as shown in Fig. [3] and other details are given therein[ए]].

- Fiber Detectors: Due to the indirect detection of diffused photons from the wall of container in cone type detectors, there are significant loss of photons[ए2]. In addition, the geometry of the detector also resulted in a large spatial non-uniformity in the signal across the detector and a variation $\sim 30 \%$ [ [12] is observed in photon yield from the center to the edge of the detector. In order to overcome these limitations, we have developed the modified configuration of scintillator detectors by using wavelength shifting (WLS) fibers and the advantages of using WLS are mentioned in the work of P.K. Mohanty et al.[ए2]. The saturation of the PMTs encountered at very high particle densities in the earlier cone detectors, limited their use over a wider range of primary cosmic ray energies. To overcome the limitation of saturation of PMT, the WLS fiber detectors have been designed with two PMTs as shown in Fig. 田. The main design goal for the new detector is to efficiently record shower particles over a large dynamic range ( 1 - 5000 particles), to cover a wide range in the energy spectrum of the primary cosmic rays from $10^{13} \mathrm{eV}$, where the particle density at the core is rather small to $10^{17} \mathrm{eV}$ where densities may reach several thousand particles $\mathrm{m}^{-2}$. Efficient detection of a single or a few particles is ensured by designing a detector with high photon yield and good spatial uniformity. The high photon yield also implies that the PMT may be operated 
at a relatively lower voltage, thereby reducing the noise and increasing its life. The PMTs are typically operated at a gain of $\times 10^{6}$, which is within the recommended operating region specified by the manufacturer. However, at high particle densities, the anode current become very large and the PMT response become non-linear. For the ETL-9807B PMT used here, the response becomes non-linear at a peak anode current of 50-150 mA. This translates into the onset of the non-linearity at $\gtrsim 50$ particles and of saturation at $\sim 100-300$ particles. This introduces error in the estimation of the various shower parameters. Although, one can use the signal from an earlier stage of the dynode chain to avoid saturation, we prefer to employ a second PMT to enhance the dynamic range of our detectors. The configuration of 2-PMT detectors is explained in[ए2]. Presently, there are 105 numbers of 2-PMT WLS fiber detectors have been deployed in the array.

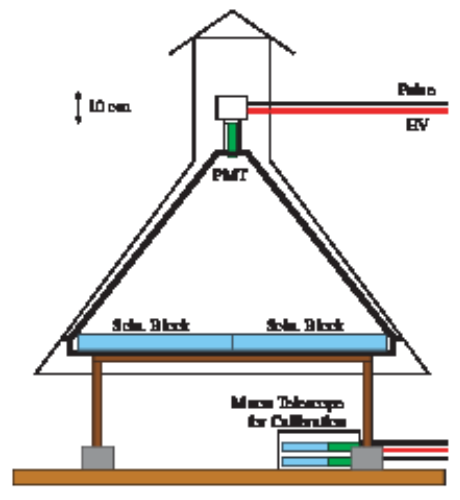

Figure 3: Schemetic of a scintillator detector, including the padels used for calibration

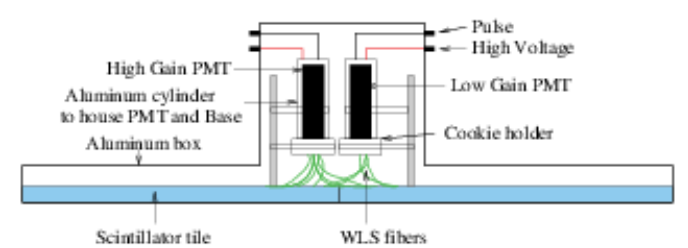

Figure 4: Cross sectional view of 2-PMT Fiber Detector

\section{Calibration of scintillator detectors}

An efficient detector system should provide a good separation between PMT noise and signal of charged particles in EAS. As shown in Fig. [3 the arrangement of calibrating a scintillator detectors. A small muon telescope made of two independent scintillator paddles (each $15 \times 15 \mathrm{~cm}^{2}$ in area and $5 \mathrm{~cm}$ in thickness) placed inside an aluminum box with a vertical separation of $5 \mathrm{~cm}$ between them. Each being fully sensitive to the passage of minimum-ionizing particles, a 2-fold $100 \mathrm{~ns}$ coincidence between the two scintillators selects almost all muons with zenith angle $\theta \lesssim 50^{\circ}$. Most of these muons pass through the scintillator detector located above the muon telescope, thus providing the distribution of integrated-charge for the passage of minimum-ionizing particles[远]. Typical single-particle response for cone type and 2-PMT WLS fiber detectors in terms of the distribution of integrated charge (ADC counts) is shown in Fig. $5 \mathrm{a}$ and Fig. $5 \mathrm{~b}$ respectively.

The high voltage for each detector PMT has been adjusted to give a most probable ADC value of about 40 counts for a minimum-ionizing particle as shown in top panel of Fig. 6 , there were higher values were used earlier of single particle gain[ए], ㅁ]. Since there are two PMTs involved for enhancing the range of the detection of particle densities, this is achieved after operating one of the PMTs at relatively lower operating voltage called as low-gain PMT (LG-PMT) as compared 


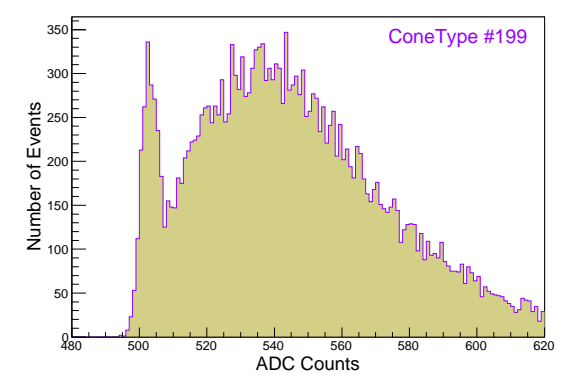

(a) Cone Det. Single Particle ADC Distribution

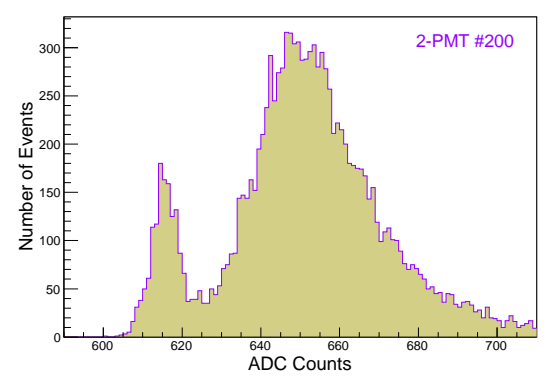

(b) 2-PMT Det. Single Particle ADC Distribution

Figure 5: Single particle ADC distributions

to the other PMT called as high-gain PMT (HG-PMT). Therefore, in this case, the most probable ADC count value is quite small for minimum ionizing particle. In addition, to enhance the linearity of PMT less number of WLS fibers are synced to the LG-PMT. The signal of single particle from LG-PMT is very fable and can not be separated with the PMT noise (pedestal signal) and therefore, not possible to calibrate it for single particle calibration as can be seen from middle panel of Fig. 6 . Therefore, an indirect method have been adopted to calibrate LG-PMT from the EAS data. After subtracting concurrent pedestal values from ADC counts, a ratio of high- and low-gain PMT ADC (HLR) is calculated in the steps of 50 ADC counts of the high gain PMT. The mean ratio 'HLR' is plotted against the high-gain PMT (HG-PMT) ADC counts as shown in bottom panel of Fig. 6 . The flat or horizontal region in bottom panel of Fig. 6 corresponds to the common linear region for both the HG- and LG-PMTS. This flat region is fit to a straight line parallel to the X-axis and its intercept on the Y-axis provides, the ratio of the gains of the HG- and LG-PMTs in the detector, whereas Fig. $\square$ shows the daily variation of calculated gain ratio variation of one of the detector. At the lower particle densities, the LG-PMT becomes relatively insensitive. Therefore, the ratio of the ADC counts of HG- to the LG-PMT shows a reduction at small particle densities.
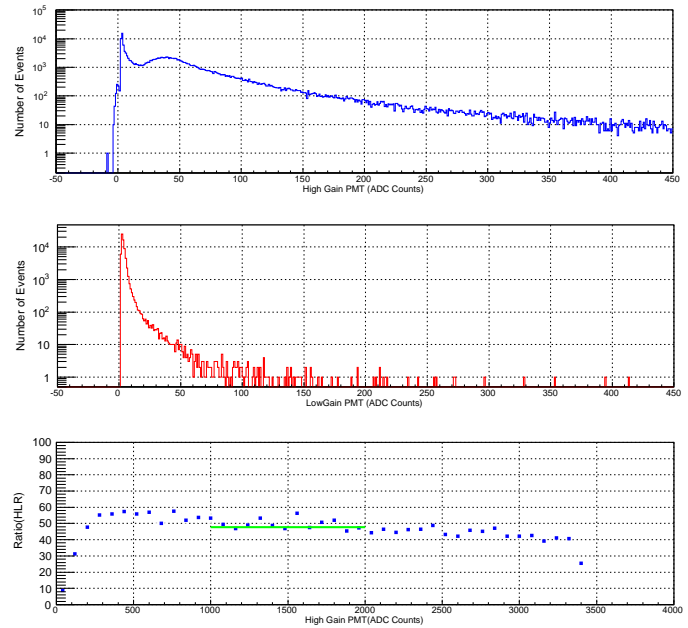

Figure 6: Gain Ratio of high- and low-gain PMT

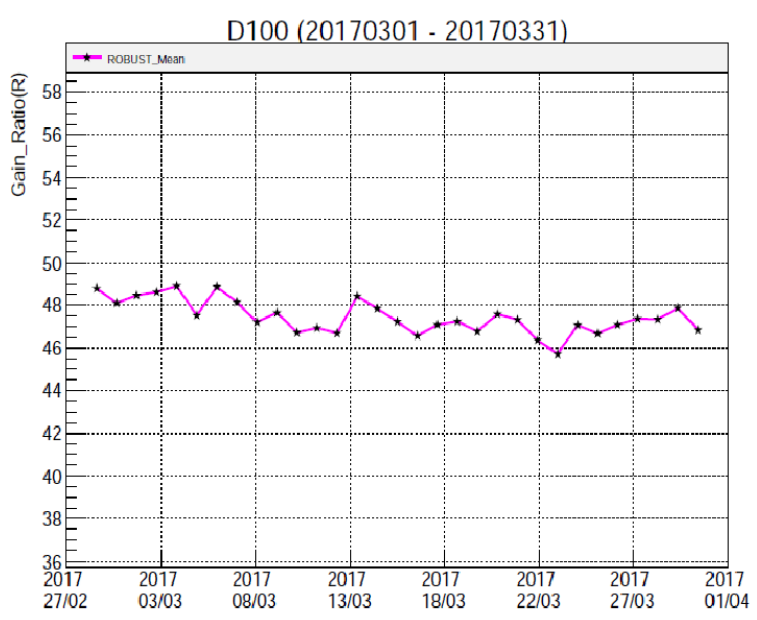

Figure 7: Gain Ratio Variation 


\section{Calculation of Particle Density}

Using the single particle calibration for the HG-PMT, and the extracted single particle gain as described in the last section for the LG-PMT, the integral particle density spectrum observed by GRAPES-3 experiment has been obtained. The integral particle density spectra for detectors 50, 110, 198 and 284 are shown in Fig. [8. For the LG-PMTs, the density spectrum shows stable power law distribution above 50 particles $\mathrm{m}^{-2}$, as the value of the density is rather uncertain below this threshold. In the case of the HG-PMT the response becomes non-linear above a density of 50 particles $\mathrm{m}^{-2}$ and it saturates in the region of $(100-300)$ particles $\mathrm{m}^{-2}$. On the other hand, the LG-PMT response remains linear $\gtrsim 5000$ particles $\mathrm{m}^{-2}$ and no saturation is observed. Thus the combination of two PMTs allows the dynamic range of the fiber detector to be extended from 1 to 5000 particles $\mathrm{m}^{-2}$. For most of the detectors the gain ratio of the HG- and the LG-PMTs, is adjusted to be (45 - 55) with a mean value of 50. For determining the density spectrum of the particles in the air showers, we have used the data from the HG-PMTs for the densities below 50 particles $\mathrm{m}^{-2}$ and the LG-PMTs for the densities above this value for the five months of uninterrupted data from 01 December to 30 April 2017.
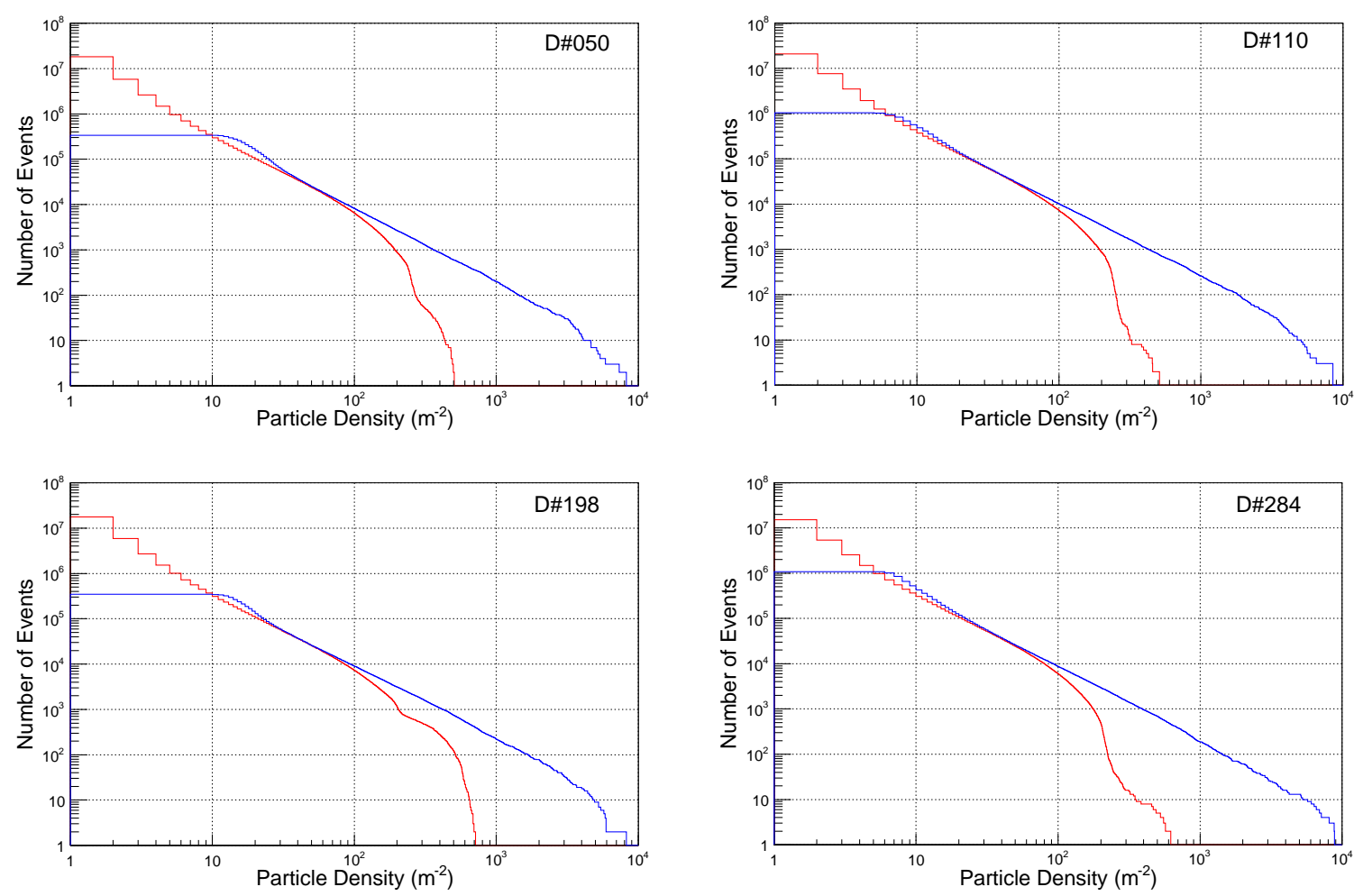

Figure 8: Particle density per $\mathrm{m}^{-2}$ for 2-PMT detectors 50, 110, 198, 284 for HG- and LG-PMT

\section{Results and Discussions}

The integral particle density spectrum measured by the 2-PMT fiber detectors has been obtained to validate the design of these new detectors. The limited dynamic range of the earlier cone 
type scintillator detectors had restricted the maximum detectable particle density to about $500 \mathrm{~m}^{-2}$. However, with the new 2-PMT detectors this range has been extended to nearly $\gtrsim 5000$ particles $\mathrm{m}^{-2}$ allowing meaningful measurements of density spectrum of particles in the EAS and expected for the composition study in the range of $10^{13} \mathrm{eV}-10^{17} \mathrm{eV}$.
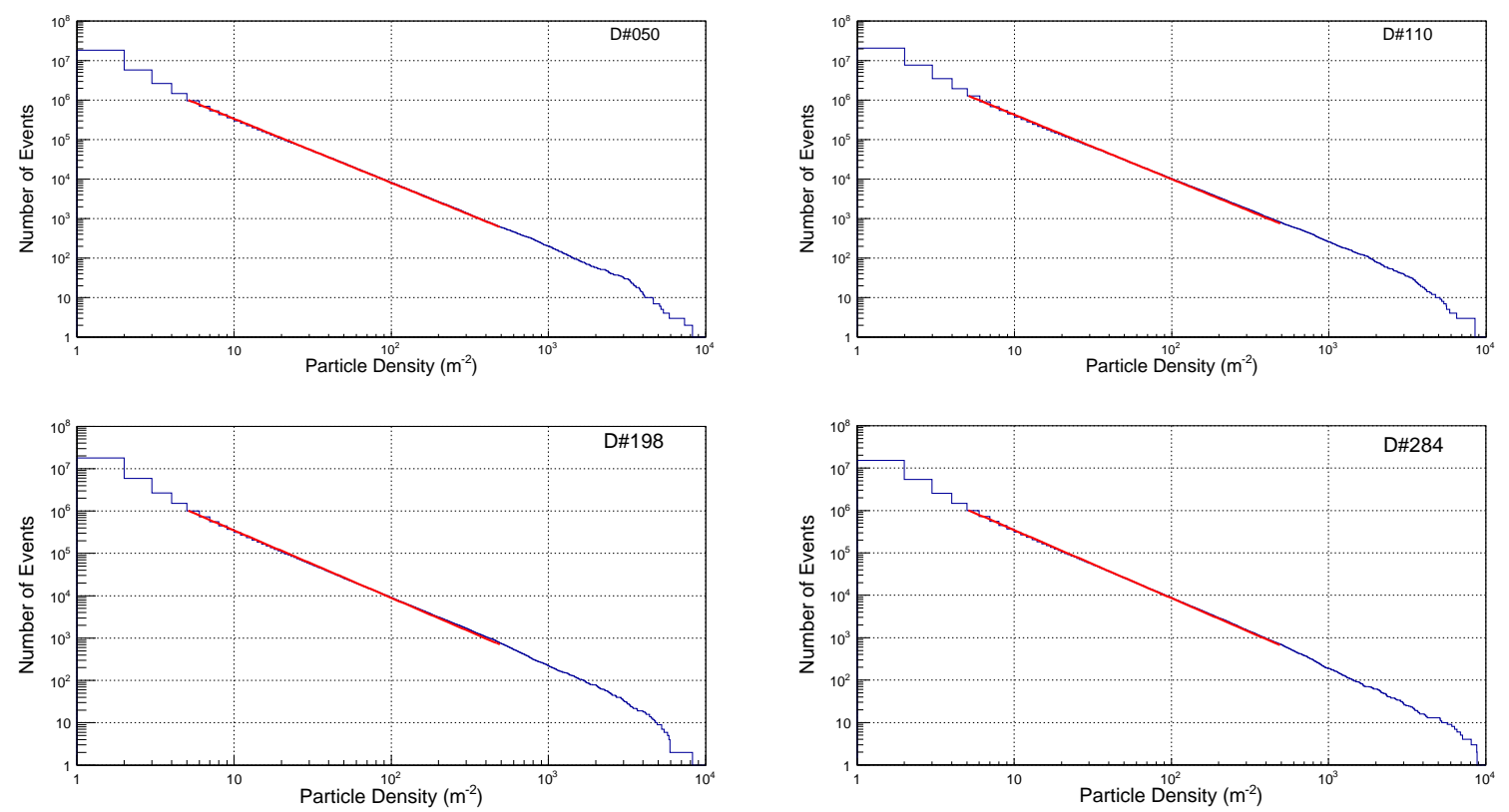

Figure 9: Particle density per $\mathrm{m}^{-2}$ for 2-PMT detectors 50, 110, 198, 284 for HG- and LG-PMT

The integral particle density spectra for four 2-PMT fiber detectors namely 50, 110, 198, 284, respectively are shown in Fig. 8 . All four spectra display a power law behavior and a fit for the density range from 5 to 500 particles $\mathrm{m}^{-2}$, yields a spectral slope of $\sim 1.61$, consistent with the earlier measurements[[13, [4]]. These values are also close to the slope of the energy spectrum of the primary cosmic rays, as has been pointed out in the past[[13]]. The nearly identical power law spectral index for all four detectors, indicates that the 2-PMT detectors are performing with a reasonably linear response, free from the effects of any saturation.

\section{Conclusions}

The performance of the 2-PMT WLS fiber detectors are compared with the cone type single PMT detectors and have shown significant improved signal to noise separation. The dynamic range of 2-PMT fiber detectors for the measurement of the particle density has increased to $\gtrsim 5 \times 10^{3}$ particles $\mathrm{m}^{-2}$. This enhanced dynamic range covers the particle densities encountered near the cores of the extensive air showers of energy well above the 'knee' in the spectrum. Presently, 105 numbers of 2-PMT fiber detectors are functional in the array and more are being continuously added. The integral particle density spectrum of the 2-PMT fiber detectors displays a spectral slope of $\gamma \sim 1.61$. This value is consistent with the value reported earlier in the literature. 


\section{Acknowledgment}

We thank D.B. Arjunan, K. Manjunath and the colleagues are available in control room for their help in testing, installation and operation of the detectors and the electronic module. We thank the group members who have given their valuable suggestions in the installation and the calibration of 2-PMT detectors.

\section{References}

[1] K. Asakimori, et al., Astrophys. J. 502 (1998) 278.

[2] M. Boezio et al., Astropart. Phys. 19 (2003) 583.

[3] Haino et al., Phys. Lett. B 594 (2004) 35.

[4] Y.S. Yoon et al., The Astrophys. J. 728 (2011) 122.

[5] M. Aguilar et al., Phys. Rev. Lett. 114, 171103 (2015).

[6] O. Adriani et al., Science 332 (2011) 69.

[7] J. Alcaraz et al., Phys. Lett. B 494 (2000) 193.

[8] H. Tanaka, et al., J. Phys. G: Nucl. Part. Phys. 39 (2012) 025201.

[9] A.A. Radu, et al., Romanian Reports in Physics, Vol. 60, No. 1, P. 4555, 2008.

[10] S.K. Gupta et al., Nuclear Instruments and Methods in Physics Research A 540 (2005) 311323.

[11] S.C. Tonwar, et al., Nuclear Physics B (Proc. Suppl.) 151 (2006) 477480.

[12] P.K. Mohanty et al., Astroparticle Physics 31 (2009) 2436.

[13] K. Greisen, Ann. Rev. Nucl. Sci. 10 (1960) 63.

[14] I. Katsumata, J. Phys. Soc. Jpn. 19 (1964) 800. 